\title{
Sedation in the intensive care setting
}

This article was published in the following Dove Press journal:

Clinical Pharmacology:Advances and Applications

24 October 2012

Number of times this article has been viewed

\section{Christopher G Hughes \\ Stuart McGrane \\ Pratik P Pandharipande \\ Vanderbilt University School of Medicine, Nashville, TN, USA}

Correspondence: Pratik P Pandharipande Vanderbilt University School of Medicine, I2II 2I st Ave South, 526 MAB,

Nashville, TN 37212, USA

Tel + I 6153436268

Fax +l 6153436272

Email pratik.pandharipande@vanderbilt.edu

\begin{abstract}
Critically ill patients are routinely provided analgesia and sedation to prevent pain and anxiety, permit invasive procedures, reduce stress and oxygen consumption, and improve synchrony with mechanical ventilation. Regional preferences, patient history, institutional bias, and individual patient and practitioner variability, however, create a wide discrepancy in the approach to sedation of critically ill patients. Untreated pain and agitation increase the sympathetic stress response, potentially leading to negative acute and long-term consequences. Oversedation, however, occurs commonly and is associated with worse clinical outcomes, including longer time on mechanical ventilation, prolonged stay in the intensive care unit, and increased brain dysfunction (delirium and coma). Modifying sedation delivery by incorporating analgesia and sedation protocols, targeted arousal goals, daily interruption of sedation, linked spontaneous awakening and breathing trials, and early mobilization of patients have all been associated with improvements in patient outcomes and should be incorporated into the clinical management of critically ill patients. To improve outcomes, including time on mechanical ventilation and development of acute brain dysfunction, conventional sedation paradigms should be altered by providing necessary analgesia, incorporating propofol or dexmedetomidine to reach arousal targets, and reducing benzodiazepine exposure.
\end{abstract}

Keywords: fentanyl, propofol, dexmedetomidine, Behavioral Pain Scale, Richmond AgitationSedation Scale, Sedation-Agitation Scale, Confusion Assessment Method for the ICU

\section{General principles of sedation in the intensive care setting}

Ensuring patient comfort and safety is a universal goal that has been endorsed by national medical societies and oversight bodies. In critically ill patients, pain and anxiety contribute to an already prominent sympathetic stress response that includes increased endogenous catecholamine activity, increased oxygen consumption, tachycardia, hypercoagulability, hypermetabolism, and immunosuppression. ${ }^{1}$ Furthermore, unrelieved pain and anxiety can lead to severe agitation and the removal of lifesaving medical devices (eg, endotracheal tubes and intravascular lines), placing both the patient and health care providers at risk. This may also contribute to significant physical and psychological stress during the acute event and in the future, when long-term consequences such as posttraumatic stress disorder (PTSD) may develop. ${ }^{2}$ Analgesia and sedation, therefore, are administered to provide patient comfort and ensure patient safety while decreasing the stress response; however, oversedation occurs frequently and is associated with longer time on mechanical ventilation and in the intensive care unit (ICU), greater need for radiological evaluations of mental status, and higher 
probability of developing brain dysfunction. ${ }^{3-5}$ To optimize patient care, safety, and comfort while minimizing the negative outcomes associated with pharmacotherapy, health care professionals must achieve the right balance of analgesic and sedative drug administration. A wide discrepancy exists, however, in the approach and administration of these medications due to patient and provider variability, bias, and regional preference. ${ }^{1}$

Patients in the ICU have unpredictable pharmacokinetics and pharmacodynamics secondary to hemodynamic instability, drug interactions, altered protein binding, and impaired organ function. This increases the difficulty of achieving benefit from analgesic and sedative medications without harm from their associated complications. Drug accumulation from continuous infusions, redistribution, and tachyphylaxis also confound the utilization of sedatives, necessitating techniques to prevent systemic drug accumulation. Thus, to develop the best treatment strategy for analgesia and sedation, the specific medical condition necessitating treatment must be recognized and continually reevaluated. Thereafter, objective routine assessments of pain, arousal, and acute brain dysfunction (eg, delirium and coma) are necessary to guide the adjustment of goal-directed therapeutic targets that change with the medical condition of the patient. ${ }^{1}$

\section{Analgesia}

Mechanical ventilation, invasive monitoring, preexisting diseases, nursing interventions, and medical procedures are only a few sources of discomfort commonly experienced in the ICU. Insufficient pain relief can contribute to increased stress response, deficient sleep, disorientation, anxiety, delirium, and PTSD. ${ }^{2,6,7}$ Unfortunately, pain is often undertreated secondary to concerns about the adverse effects of medications (eg, respiratory depression and hemodynamic compromise), addiction potential of opioids, and lack of proper knowledge in pain assessment and treatment. ${ }^{1}$

\section{Assessment of pain}

Routine monitoring that includes intensity, quality, and location of the pain has been associated with lower analgesic and sedative utilization and decreased time on mechanical ventilation. ${ }^{8}$ The Behavioral Pain Scale is an example of a validated tool for assessing pain in ICU patients unable to communicate, ${ }^{9}$ and pain should be evaluated as part of all patients' vital signs, including those sedated and mechanically ventilated.

\section{Analgesia management}

Nonpharmacologic methods for managing pain in the ICU include patient repositioning, lumbar support, injury stabilization, removal of noxious or irritating stimuli, and application of heat or cold. When nonpharmacologic methods are insufficient to control pain, provision of analgesia by regional or systemic therapy is indicated.

\section{Regional analgesic therapy}

Regional analgesic therapies provide analgesia for specific areas of the body without the systemic effects of intravenous analgesics. Blockade of an individual nerve or nerve plexus may provide relief of pain localized to one extremity, and this targeted action can be prolonged by placement of a peripheral nerve catheter. ${ }^{10}$ Pain due to thoracic or upper abdominal trauma or surgery can be managed by intercostal nerve blocks, which can improve respiratory mechanics to reduce the risk of pulmonary compromise. ${ }^{11}$ Paravertebral blocks are useful for managing pain related to unilateral thoracic or abdominal procedures and traumatic rib fractures. ${ }^{12}$ Epidural analgesia has become increasingly popular for the management of pain from thoracic, abdominal, or lower extremity operative procedures, providing bilateral analgesia in specific dermatomes. Multiple studies examining epidural analgesia have shown reduced morbidity after major surgery, including improved pulmonary and intestinal function, ${ }^{13}$ but epidural analgesia has not been shown to reduce mortality or length of stay despite improving pulmonary function in a meta-analysis of traumatic rib fracture patients, a commonly prescribed indication. ${ }^{14}$ Bupivacaine and ropivacaine are the local anesthetics most commonly utilized for regional analgesic therapy; however, opioids, clonidine, dexamethasone, and other pharmaceutical adjuncts are also utilized. While these procedures are useful adjuncts to decrease exposure to side effects of potent analgesics, they are not without risk. In the ICU, these regional techniques likely have higher risk of failure, infection, bleeding, neuronal injury, pneumothorax, and hemodynamic compromise due to the patients' critical illness; therefore, they should only be performed by specially trained clinicians.

\section{Systemic analgesic therapy}

Systemic analgesics should be administered as part of a goaldirected analgesia and sedation protocol. Systemic therapies include acetaminophen and nonsteroidal anti-inflammatory drugs such as ketorolac, but the most commonly used 
analgesics in the ICU are opioids secondary to their analgesic and sedative properties. Although they are the mainstay of analgesia in the ICU, opioids have a number of adverse effects. Respiratory depression is commonly seen and often enhanced by co-administration of additional sedative agents. Hypotension may result from decreased sympathetic tone or vasodilation from histamine release. Other side effects include decreased gastrointestinal motility, pruritus, flushing, urinary retention, and delirium. Consequently, nonopioid analgesics should be considered for treatment of low acuity pain or as adjuncts to decrease opioid exposure to preserve mental status and pulmonary function while reducing additional side effects.

\section{Morphine and hydromorphone}

Morphine, hydromorphone, fentanyl, and remifentanil are frequently used opioids in the ICU. Morphine and hydromorphone are most often utilized as intermittent intravenous (IV) injections. Morphine is often given in doses of 2-5 mg IV every 5-15 minutes until the pain is controlled, followed by similar doses on a scheduled basis every 2-4 hours. Morphine is characterized by hepatic metabolism and renal excretion with intermediate volume of distribution. Therefore, its effects can be prolonged in patients with renal or hepatic impairment or obesity. ${ }^{15}$ Hydromorphone is a more potent congener of morphine with similar pharmacokinetic and pharmacodynamic profiles. ${ }^{15}$ Its lack of histamine release and decreased incidence of central nervous system side effects make it a useful alternative to morphine, with typical dosing ranges of $0.2-1.0 \mathrm{mg}$ IV every $10-15$ minutes until pain is controlled, followed by similar doses every 2-4 hours. Unlike morphine, hydromorphone does not have active metabolites; thus, it has an improved safety profile in patients with renal disease.

\section{Fentanyl and remifentanil}

Fentanyl is a synthetic opioid with a rapid onset (5-15 minutes) and a short duration of action (30-60 minutes). It is easily titrateable as a continuous infusion secondary to its short half-life. In general, loading doses of 25-100 $\mu \mathrm{g}$ of fentanyl are given every 5-10 minutes until the pain is controlled, followed by infusion rates of $25-250 \mu \mathrm{g} / \mathrm{h}$. It has a large volume of distribution secondary to its lipophilicity, while its clearance correlates most closely with pharmacokinetic mass (similar to lean body mass); therefore, significant drug accumulation and a prolonged context sensitive half-life can occur with prolonged infusions. ${ }^{16}$ However, because it causes less histamine release than morphine and does not undergo renal elimination, it is the preferred opioid analgesic in hemodynamically unstable patients or those with renal insufficiency. ${ }^{1}$

Remifentanil, a derivative of fentanyl, is unique as an opioid secondary to its metabolism by nonspecific blood and tissue esterases. It is utilized primarily as an infusion $(0.05-2.00 \mu \mathrm{g} / \mathrm{kg} / \mathrm{min})$ and has an elimination half-life of less than 10 minutes regardless of infusion duration. Dosing regimens for the infusion should be based on ideal body weight or lean body mass, ${ }^{17}$ and hypotension and bradycardia are the most common side effects seen with remifentanil administration. Importantly and secondary to its ultra-short half-life, supplemental analgesic medication is required at the conclusion of a remifentanil infusion.

\section{Selection of opioid therapy}

The selection of an opioid for systemic analgesia has traditionally depended on the pharmacology of the specific opioid and the likely required duration. Unfortunately, few comparative trials have been performed in critically ill patients. Remifentanil provided better outcomes than morphine with regards to time at optimal arousal level, necessity of supplemental sedation, duration of mechanical ventilation, and extubation time in one randomized double blind study. ${ }^{18}$ Meanwhile, remifentanil and fentanyl have displayed equal efficacy in achieving sedation goals with no difference in extubation times. ${ }^{19}$ Patients receiving fentanyl required more breakthrough sedatives but experienced less pain after extubation compared with patients receiving remifentanil. ${ }^{19}$ Higher cost and reports of withdrawal and hyperalgesia have limited the widespread utilization of remifentanil for analgesia in the ICU. In general, fentanyl's rapid onset, short duration of action, relatively short half-life, minimal histamine release, lack of renal elimination, and easy titration as a continuous infusion make it the opioid of choice in hemodynamically unstable patients. ${ }^{1}$

With regards to acute brain dysfunction outcomes, the literature is inconsistent. In a prospective cohort study of elderly hip fracture patients, the patients who received higher morphine equivalents per day were less likely to develop delirium than patients who received less analgesic medications. ${ }^{6}$ Additionally, studies in critically ill trauma and burn patients have also reported on the beneficial effects of morphine and methadone in reducing the development of delirium. ${ }^{20,21}$ However, meperidine and morphine have been 
positively associated with increased risk for delirium..$^{22,23}$ Thus, providing systemic analgesia with opioids to patients in pain may be protective of acute brain dysfunction, while excessive administration to achieve sedation may be detrimental.

\section{Sedation}

Sedative medications are commonly prescribed within the ICU environment primarily for the treatment of agitation and anxiety, which themselves may be caused by many different conditions (eg, dyspnea, delirium, mechanical ventilation, lack of sleep, and untreated pain). The appropriate use of sedatives can facilitate patient care and contribute to patient safety; however, their use is associated with both short- and long-term negative patient outcomes, including prolonged mechanical ventilation and cognitive dysfunction. ${ }^{3,4,24}$ It is important, therefore, to define the indication for sedation, as this may affect the sedative selection and help determine the endpoint for sedative utilization.

\section{Arousal monitoring}

There are many ICU arousal scales which are used to provide goal directed therapy individualized to the patient. The most widely used arousal scales are the Richmond Agitation-Sedation Scale and the Riker Sedation-Agitation Scale. ${ }^{25,26}$ When used appropriately, these scales can provide a therapeutic target, which can lead to decreased dosing of sedative medications and decreased time on mechanical ventilation. ${ }^{27}$ However, arousal assessment is part of the neurological examination of all critically ill patients and should not be exclusively linked to sedative drug administration. It is important to recognize that the arousal scales are not applicable when the patient is being administered neuromuscular blocking drugs, and consideration should be given to the use of the Bispectral Index monitor in those instances.

\section{Delirium monitoring}

Delirium is an acute fluctuating change in mental status characterized by inattention and altered levels of consciousness that is now considered to be a presentation of brain organ dysfunction. Prevalence within the ICU can be up to $80 \%$, and it can lead to long-term cognitive dysfunction. ${ }^{28}$ The pathogenesis of delirium is not fully appreciated, and there are many proposed hypotheses including inflammatory changes, impaired oxidative metabolism, neurotransmitter disturbances, and alterations in amino acid precursors. ${ }^{29-31}$ Delirium is associated with the use of sedative medications and contributes to increased mortality, morbidity, hospital length of stay, and cost. ${ }^{4,32-36}$ The presence of delirium, therefore, should be assessed using validated instruments such as the Intensive Care Delirium Screening Checklist or the Confusion Assessment Method for the ICU as part of the routine neurological examination of critically ill patients. . $^{37,38}$

\section{Sedation protocols}

Sedation protocols are commonplace within ICU environments and provide a structured framework that guides sedative administration and monitoring. Their use alone has been associated with significantly improved patient outcomes. ${ }^{39-41}$ Nurses, secondary to their more consistent presence at the patient's bedside, are the most appropriate providers to implement the sedation protocols, and in fact, protocols administered by the nursing staff have been shown to improve patient outcomes. ${ }^{39,42}$

Key elements of sedation protocols should include arousal monitoring instruments, sedative dosing instructions, spontaneous awakening trials that are linked to spontaneous breathing trials, and early mobilization therapy. Spontaneous awakening trials (daily interruption of sedation) have been shown to reduce duration of mechanical ventilation, decrease ICU length of stay, and decrease the incidence of PTSD. ${ }^{43,44} \mathrm{~A}$ multicenter randomized controlled trial that combined the use of spontaneous awakening trials with spontaneous breathing trials (the ABC study) showed that this strategy decreased time on mechanical ventilation, reduced ICU and hospital lengths of stay, and improved 1-year survival. ${ }^{45}$

Despite numerous studies demonstrating that deep sedation is not required in the majority of ICU patients and that lighter sedation goals improve outcomes, many providers have been hesitant to implement these techniques due to concern for patient safety and the belief that patients would be more likely to develop long-term psychological issues without deep sedation during their ICU stay. The ABC trial, however, showed no difference in the rate of re-intubation between the control and intervention groups, and studies incorporating daily wake up trials have shown no increase in the incidence of PTSD. ${ }^{44,46}$ In fact, sedative utilization (in particular lorazepam) has been associated with PTSD, and the number of days of sedation has been correlated with PTSD and depression. ${ }^{24,47}$ While unpleasant memories of their ICU course may contribute to psychological distress in survivors, ${ }^{7,48}$ PTSD is more often related to having delusional and not factual memories of the ICU stay. ${ }^{49,50}$ Additionally, patients with recall of their ICU stay have less cognitive 
dysfunction than patients with complete amnesia, further emphasizing that deep sedation may have prolonged neuropsychological and cognitive effects. ${ }^{51}$

It is advisable that each ICU develop a local sedation protocol that takes into account current research, patient characteristics, and local evidence. Readers are also encouraged to visit the Society of Critical Care Medicine's website (www.SCCM.org) for up-to-date sedation guidelines and other valuable resources. Prior to implementation, all staff involved in administering the protocol should be trained in its application and opportunity given for modification when necessary.

\section{Pharmacology management}

Providers should recognize that sedative medications are considered part of a multimodal approach to ensuring patient comfort and safety. Important aspects also include providing analgesia, maintenance of a normal day-night cycle, patient positioning, and appropriate mechanical ventilation strategies. It must also be appreciated that sedatives should only be considered once pain has been adequately treated - the concept of analgesia-based sedation or analgosedation. Once analgesia has been obtained, sedative medications can be utilized to reach arousal targets when needed. An empiric protocol (Figure 1) for the management of pain, sedation, and delirium is provided as a reference.

\section{Selection of a sedative regimen}

The ideal sedative will be inexpensive, have minimal respiratory depression, elimination independent of organ function, short context sensitive half-life, and no active metabolites. Unfortunately, none of the commonly used sedatives fulfill all these criteria, and practitioners should be aware of their limitations when choosing a sedative medication. The most common sedative medications used within the ICU are propofol, dexmedetomidine, and benzodiazepines, with other agents such as clonidine, ketamine, volatile anesthetics, and neuromuscular blockers used as adjunct therapies.

Importantly, the duration of sedative medication administration has been shown to correlate with the duration of mechanical ventilation, and the consistent theme throughout many sedation studies is that efforts should be made to minimize the total dose of sedative by using the minimum effective dose, daily interruption of sedation, and infusions for the shortest time required. ${ }^{3,43}$ Furthermore, there is growing literature that favors the avoidance of benzodiazepines for sedation in the ICU in favor of propofol, dexmedetomidine, or analgosedation regimens.

\section{Propofol}

Propofol is a diisopropylphenol anesthetic and a $\gamma$-aminobutyric acid (GABA) agonist. It has proven utility as a sedating agent in the ICU due to its rapid onset (1-2 minutes) and short duration of action (2-8 minutes). It is typically given as a bolus injection of 40-100 $\mathrm{mg}$ IV followed by an infusion of $25-75 \mu \mathrm{g} / \mathrm{kg} / \mathrm{min}$. Its volume of distribution is large with a short distribution half-life. Emergence is related to redistribution and not metabolic clearance when used as bolus or low-dose infusion, which can be advantageous in patients with renal or hepatic dysfunction. When propofol is used as a long-term infusion and saturation of peripheral tissues occurs, emergence is more related to metabolic clearance. $^{52}$

Propofol side effects include hypotension due to vasodilation and myocardial depression, respiratory depression, and hypertriglyceridemia. The hypertriglyceridemia may either be due to the intralipid carrier or altered hepatic lipid metabolism, which can be seen with the propofol infusion syndrome (PRIS). ${ }^{53}$ PRIS is associated with increased dosage of propofol (doses $>75 \mu \mathrm{g} / \mathrm{kg} / \mathrm{min}$ or $>5 \mathrm{mg} / \mathrm{kg} / \mathrm{h}$ ), pediatric sedation, critical illness, and prolonged infusions ( $>48$ hours) and is characterized by severe lactic acidosis and rhabdomyolysis. ${ }^{53} \mathrm{~A}$ high index of suspicion is necessary for prompt recognition given the high mortality rate with PRIS, especially considering there are no specific treatments other than supportive management and discontinuation of propofol. When high dosage or prolonged infusions are being used, it is recommended to regularly monitor serum $\mathrm{pH}$, lactate, creatinine kinase, triglyceride levels, and electrocardiograms (Brugada-type changes). ${ }^{54}$

\section{Dexmedetomidine}

Dexmedetomidine is an alpha-2 receptor agonist whose site of action includes presynaptic neurons in the locus ceruleus and spinal cord. It causes sedation and analgesia without significant respiratory depression. Sedation is often initiated with a bolus of $1 \mu \mathrm{g} / \mathrm{kg}$ over 10-20 minutes, followed by an infusion of $0.2-0.7 \mu \mathrm{g} / \mathrm{kg} / \mathrm{h}$. Studies have shown safety with doses up to $2 \mu \mathrm{g} / \mathrm{kg} / \mathrm{h}$, although with increased incidence of bradycardia (most common side effect) and hypotension. ${ }^{55}$ Hypertension can also result from stimulation of postjunctional alpha-2 receptors located on arterial and venous smooth muscle; this is more likely to be seen with bolus dosing and has led numerous providers to routinely avoid 


\section{Analgesia/sedation protocol for mechanically ventilated patients}

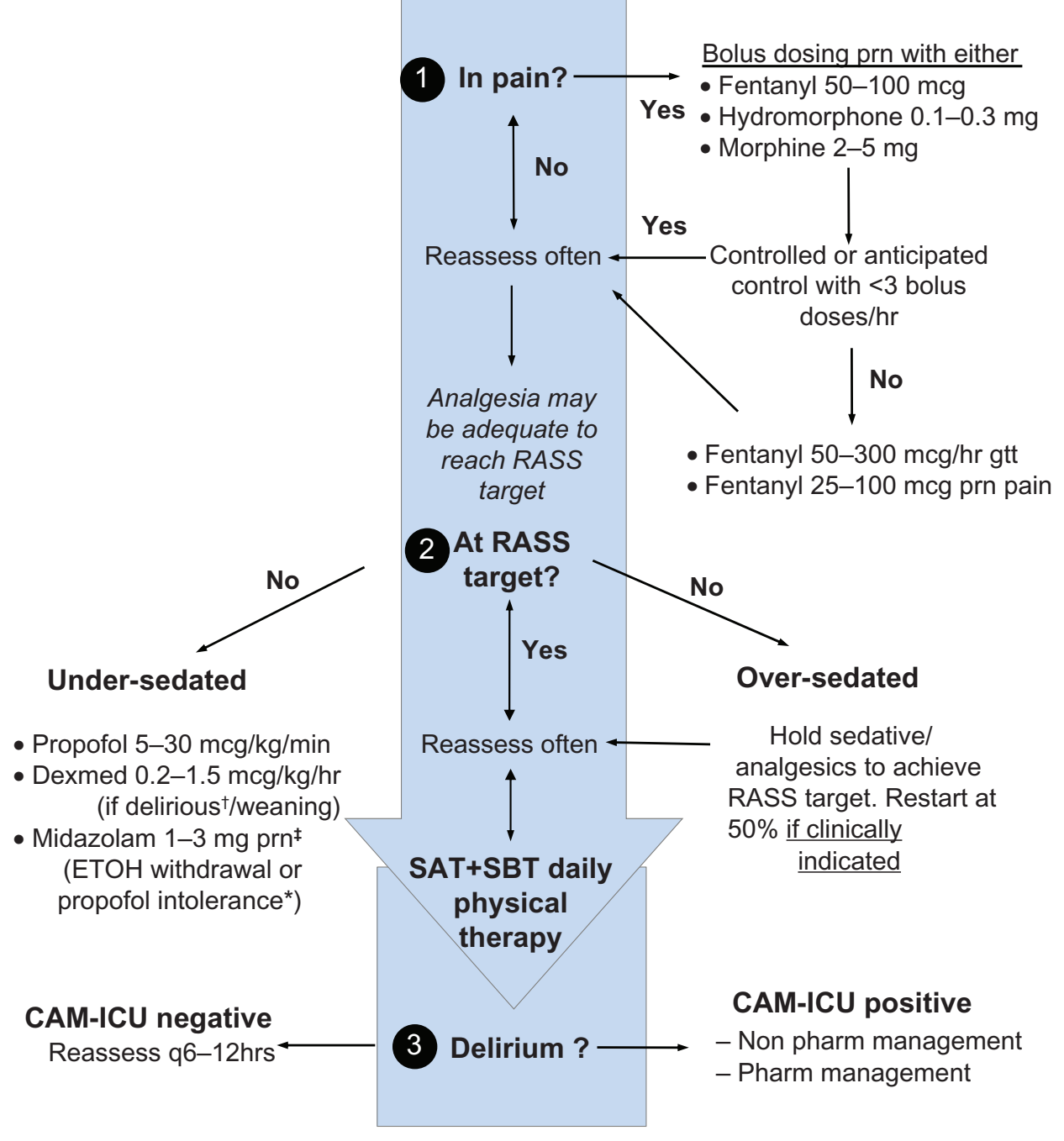

Figure I Empiric sedation protocol.

With permission from www.icudelirium.org.

Notes: $¥$ Midazolam I-3 mg/hr gtt rarely if $>2$ midaz boluses/hr and propofol intolerance. *Propofol intolerance refers to propofol infusion syndrome, hemodynamic instability, increasing CPK $>5000 \mathrm{IU} / \mathrm{L}$, triglycerides $>500 \mathrm{mg} / \mathrm{dl}$ or use $>96 \mathrm{hrs}$.

Abbreviations: CAM-ICU, Confusion Assessment Method for the Intensive Care Unit; Dexmed, dexmedetomidine; ETOH, ethanol; gtt, infusion; pharm, pharmacological; prn, as needed; RASS, Richmond Agitation-Sedation Scale; SAT, spontaneous awakening trial; SBT, spontaneous breathing trial

bolus dosing in the ICU. Dexmedetomidine is metabolized by the liver, and patients with severe liver disease require lower dosing, whereas there is no need for dose adjustment in those with renal dysfunction. ${ }^{56}$

\section{Benzodiazepines}

Benzodiazepines have been used for sedation for many years within the ICU setting with midazolam, lorazepam, and diazepam being the most commonly utilized agents. They are GABA agonists metabolized in the liver to active metabolites (lorazepam being the exception with no active metabolite).
These metabolites can lead to prolongation of their sedative effects, especially in the presence of renal failure. The use of lorazepam is limited by the fact it is dissolved in propylene glycol, which can accumulate to produce metabolic acidosis and renal dysfunction. ${ }^{57}$

Despite the widespread use of benzodiazepines for sedation in the ICU, there is a growing body of evidence that shows that they are associated with poorer patient outcomes, including increased brain dysfunction, time on mechanical ventilation, and ICU length of stay. ${ }^{4,58-62}$ Their use is starting to be curtailed in the ICU, and the authors expect this will 
continue over the upcoming years with increased utilization of propofol and dexmedetomidine as the benzodiazepines become second line agents. ${ }^{63}$ Benzodiazepines, however, remain the drugs of choice for the treatment of delirium tremens (and other withdrawal syndromes) and seizures.

\section{Comparative studies of sedation regimens}

Analgosedation

Analgesia-based sedation is a concept that has been around for many years but which has had resurgence with lighter sedation techniques and the development of opioid medications with rapid onset and offset. In a randomized controlled study comparing analgesia only (remifentanil with propofol rescue) versus analgesia and sedation (titrated propofol or benzodiazepine infusion with as-needed opioid), the analgesia only group had decreased ICU length of stay, more days without mechanical ventilation, and improved SedationAgitation Scores. ${ }^{64}$ This is consistent with other multicenter trials comparing analgesia-based regimens versus sedative regimens that demonstrated analgesia-based regimens can shorten the duration of mechanical ventilation. ${ }^{65,66}$ A more recent single-center randomized controlled trial compared the use of a morphine-based protocol versus sedation with propofol and similarly found shorter times on mechanical ventilation in the intervention (morphine only) group. ${ }^{67}$ It is important to note that while approximately $80 \%$ of the patients in the intervention group were managed with morphine alone, the ICU had 1:1 nursing ratios and other personnel available to reassure patients. As discussed earlier, there are limited data to suggest a single superior analgesic drug for analgosedation regimens. Therefore, the decision on which analgesic agent to employ should be based upon clinical conditions and cost, with the agent titrated using validated scales.

\section{Clinical trials of sedatives}

When compared with benzodiazepines, propofol has been shown to increase duration at target arousal level, reduce cost per patient, and decrease time spent on mechanical ventilation. ${ }^{60-62,68} \mathrm{~A}$ meta-analysis comparing propofol to alternate sedation regimens for medium to long-term sedation demonstrated a decreased ICU length of stay that was significant when compared with the long-acting benzodiazepines (diazepam, lorazepam) but not when compared with the shorter-acting midazolam. ${ }^{69}$ Dexmedetomidine has been compared with benzodiazepines in multiple randomized controlled trials. The MENDS (comparator lorazepam) and SEDCOM (comparator midazolam) studies both demonstrated that patients sedated with dexmedetomidine had lower probability of developing delirium. ${ }^{58,59}$ Dexmedetomidine patients in the SEDCOM study also had decreased duration of mechanical ventilation and less tachycardia and hypertension. Subgroup analysis of the MENDS study showed improved outcomes in septic patients, including increased survival, with dexmedetomidine use. ${ }^{70}$

A study comparing dexmedetomidine and propofol sedation in post-surgical patients showed similar time at target sedation, but patients sedated with dexmedetomidine required less supplemental analgesia. ${ }^{71}$ When compared with propofol in post-cardiac surgical patients, patients sedated with dexmedetomidine had decreased use of beta blockers and epinephrine. ${ }^{72}$ A meta-analysis performed prior to the MIDEX and PRODEX studies described below suggested that dexmedetomidine use was associated with a significant reduction in ICU length of stay. ${ }^{55}$

In a recently published study, dexmedetomidine was compared to midazolam (MIDEX) and propofol (PRODEX) for light to moderate sedation in patients requiring mechanical ventilation for greater than 24 hours. ${ }^{73}$ Time at target arousal level was equivalent between dexmedetomidine and the control groups; however, more patients in the dexmedetomidine group required rescue drug than in the propofol group, and discontinuation due to lack of efficacy occurred more often in patients sedated with dexmedetomidine than in patients sedated with midazolam or propofol. Arousability, communication, and patient cooperation were improved with dexmedetomidine sedation. Dexmedetomidine reduced duration of mechanical ventilation compared with midazolam, and time to extubation was faster in the dexmedetomidine groups than either the midazolam or propofol groups. Overall, length of ICU and hospital stay and mortality were similar between groups.

The most often discussed concerns with dexmedetomidine are bradycardia and cost. While bradycardia was a common side effect that occurred in the MENDS, SEDCOM, and MIDEX studies, there were no significant differences between the comparator groups with regards to bradycardia necessitating treatment. ${ }^{58,59,73}$ Furthermore, neither bradycardia nor hypotension was significantly different between dexmedetomidine and propofol in the PRODEX study. ${ }^{73}$ With regard to cost, a post-hoc analysis of the SEDCOM study showed a significant per patient cost reduction with dexmedetomidine use. ${ }^{74}$ This is similar to the introduction of propofol to the market for ICU sedation, where the initial upfront cost of sedation is recouped by decreased mechanical ventilation or ICU length of stay. 
Future studies comparing outcomes, including cost, between propofol and dexmedetomidine are necessary to further delineate their potential advantages and disadvantages in different ICU patient populations.

\section{Adjuncts to sedative therapy Clonidine}

Clonidine is an alpha-2 agonist similar to dexmedetomidine; however, its clinical effects are altered secondary to differing affinity for the receptor. Clonidine can provide a low level of sedation and analgesia, and its main uses within the ICU tend to be for withdrawal syndromes (eg, alcohol withdrawal or rapid discontinuation of analgesic or sedative medications). ${ }^{75,76}$ The addition of clonidine to standard analgesic regimens has been shown to allow reduction of opioid dosage and may facilitate liberation from the ventilator. ${ }^{77}$ Clonidine itself should not be discontinued quickly due to the risk of rebound hypertension, and a gradual weaning plan should be in place prior to patient discharge from the ICU. ${ }^{78}$ The need for this planning is highlighted by the fact that, in some ICU cohorts, $85 \%$ of patients discharged from the hospital had potentially inappropriate medications on their discharge list, with $50 \%$ of these initially being prescribed in the ICU. ${ }^{79}$

\section{Ketamine}

Ketamine is an $N$-methyl- $D$-aspartate antagonist and should be considered an adjunctive sedative agent. It possesses analgesic properties and is often used in burn patients to facilitate opioid reduction. ${ }^{80}$ Concern for myocardial ischemia, raised intracranial pressure, delirium, and sympathetic stimulation has limited the use of ketamine for sedation. Recently, however, there has been evidence of potential neuroprotective effects with ketamine, leading some to include a recommendation that it be used in conjunction with a GABA agonist in patients with traumatic brain injury. ${ }^{81,82}$

\section{Volatile anesthetics}

Volatile anesthetic use for sedation within the ICU setting has been limited by problems with atmospheric pollution, administration, and ICU culture. ${ }^{83}$ Recently, devices have been developed to overcome many of these problems, and studies have demonstrated faster emergence with volatile agents compared with standard sedatives. ${ }^{84,85}$ Fluoride ion production is still a concern, especially if prolonged sedation is used, and the authors expect future studies to address this issue prior to volatile anesthesia becoming widespread within the ICU setting.

\section{Pharmacological paralysis}

With increasing evidence of the harm associated with deep sedation techniques, the utilization of neuromuscular blockade as an adjunct in the sedation of critically ill patients has decreased considerably. Pharmacologic paralysis remains utilized in patients with progressive respiratory failure and high peak inspiratory pressures unresponsive to conventional ventilation and in patients with postoperative open abdomens. Cisatracurium is the recommended agent for maintenance of paralysis in the critically ill secondary to its nonsteroidal benzylisoquinoline structure, Hoffman elimination, independence of hepatic or renal elimination, and lack of histamine release. A recent multicenter randomized controlled trial demonstrated that early utilization of pharmacological paralysis decreased mechanical ventilation time and mortality in patients with acute respiratory distress syndrome without a witnessed increase in muscle weakness. ${ }^{86}$ The results of this study need to be confirmed, and the ramifications with regards to sedation techniques still need to be further evaluated.

\section{Early mobilization}

The use of early mobilization within the ICU is increasing as studies have shown feasibility, safety, and an improvement in patient outcomes with this intervention. ${ }^{87,88}$ Early physical therapy has been shown to significantly decrease the rates of ICU and hospital acquired delirium, despite similar sedative usage.$^{87} \mathrm{~A}$ report has also shown that incorporation of a protocol to facilitate early mobilization decreased the use of sedative and analgesics, with a subsequent decline in the rate of delirium as well. ${ }^{89}$ Furthermore, combining daily interruption of sedation with physical and occupational therapy has been shown to lead to significant improvement in functional status at hospital discharge. ${ }^{87}$

\section{Brain dysfunction and sedation}

Associations between benzodiazepines and worse brain dysfunction outcomes have been found in medical, surgical, trauma, and burn ICU cohorts. ${ }^{4,20,21}$ As noted earlier, data on the effects of opioid analgesia are not consistent. The utilization of dexmedetomidine for sedation, however, has been shown to decrease duration of brain organ dysfunction when compared with benzodiazepines in medical and surgical ICU populations. ${ }^{58,59} \mathrm{~A}$ liberation and animation strategy focusing on the ABCDE's $[\underline{A}$ wakening and Breathing trials $(\underline{A B})$, Choice of sedation $(\underline{\mathrm{C}})$, ㄹelirium monitoring and management $(\underline{\mathrm{D}})$, and early Exercise $(\underline{E})]$ may potentially reduce the incidence and duration of acute and long-term brain dysfunction. ${ }^{90}$ 


\section{Conclusion}

Clinicians must strive to balance the necessity and benefit of sedative pharmacotherapy with the potential to negatively affect patient outcomes. By incorporating into practice a systematic management approach that follows the general principles of analgesia and sedation outlined in this paper, we can maximize patient comfort and care while reducing the likelihood and cost of iatrogenic complications.

\section{Disclosure}

The authors report no conflicts of interest in this work.

\section{References}

1. Jacobi J, Fraser GL, Coursin DB, et al. Clinical practice guidelines for the sustained use of sedatives and analgesics in the critically ill adult. Crit Care Med. 2002;30(1):119-141.

2. Kapfhammer HP, Rothenhausler HB, Krauseneck T, Stoll C, Schelling G. Posttraumatic stress disorder and health-related quality of life in longterm survivors of acute respiratory distress syndrome. Am J Psychiatry. 2004;161(1):45-52.

3. Kollef MH, Levy NT, Ahrens TS, Schaiff R, Prentice D, Sherman G. The use of continuous i.v. sedation is associated with prolongation of mechanical ventilation. Chest. 1998;114(2):541-548.

4. Pandharipande P, Shintani A, Peterson J, et al. Lorazepam is an independent risk factor for transitioning to delirium in intensive care unit patients. Anesthesiology. 2006;104(1):21-26.

5. Watson PL, Shintani AK, Tyson R, Pandharipande PP, Pun BT, Ely EW. Presence of electroencephalogram burst suppression in sedated, critically ill patients is associated with increased mortality. Crit Care Med. 2008;36(12):3171-3177.

6. Morrison RS, Magaziner J, Gilbert M, et al. Relationship between pain and opioid analgesics on the development of delirium following hip fracture. J Gerontol A Biol Sci Med Sci. 2003;58(1):76-81.

7. Rotondi AJ, Chelluri L, Sirio C, et al. Patients' recollections of stressful experiences while receiving prolonged mechanical ventilation in an intensive care unit. Crit Care Med. 2002;30(4):746-752.

8. Payen JF, Bosson JL, Chanques G, Mantz J, Labarere J. Pain assessment is associated with decreased duration of mechanical ventilation in the intensive care unit: a post hoc analysis of the DOLOREA study. Anesthesiology. 2009;111(6):1308-1316.

9. Payen JF, Bru O, Bosson JL, et al. Assessing pain in critically ill sedated patients by using a behavioral pain scale. Crit Care Med. 2001;29(12): 2258-2263.

10. Richman JM, Liu SS, Courpas G, et al. Does continuous peripheral nerve block provide superior pain control to opioids? A meta-analysis. Anesth Analg. 2006;102(1):248-257.

11. Karmakar MK, Ho AM. Acute pain management of patients with multiple fractured ribs. J Trauma. 2003;54(3):615-625.

12. Mohta M, Verma P, Saxena AK, Sethi AK, Tyagi A, Girotra G. Prospective, randomized comparison of continuous thoracic epidural and thoracic paravertebral infusion in patients with unilateral multiple fractured ribs - a pilot study. J Trauma. 2009;66(4):1096-1101.

13. Block BM, Liu SS, Rowlingson AJ, Cowan AR, Cowan JA Jr, Wu CL. Efficacy of postoperative epidural analgesia: a meta-analysis. JAMA. 2003;290(18):2455-2463.

14. Carrier FM, Turgeon AF, Nicole PC, et al. Effect of epidural analgesia in patients with traumatic rib fractures: a systematic review and metaanalysis of randomized controlled trials. Can J Anaesth. 2009;56(3): 230-242.

15. Horn E, Nesbit SA. Pharmacology and pharmacokinetics of sedatives and analgesics. Gastrointest Endosc Clin N Am. 2004;14(2): 247-268.
16. Shibutani K, Inchiosa MA Jr, Sawada K, Bairamian M. Pharmacokinetic mass of fentanyl for postoperative analgesia in lean and obese patients. Br J Anaesth. 2005;95(3):377-383.

17. Egan TD, Huizinga B, Gupta SK, et al. Remifentanil pharmacokinetics in obese versus lean patients. Anesthesiology. 1998;89(3):562-573.

18. Dahaba AA, Grabner T, Rehak PH, List WF, Metzler H. Remifentanil versus morphine analgesia and sedation for mechanically ventilated critically ill patients: a randomized double blind study. Anesthesiology. 2004;101(3):640-646.

19. Muellejans B, Lopez A, Cross MH, Bonome C, Morrison L, Kirkham AJ. Remifentanil versus fentanyl for analgesia based sedation to provide patient comfort in the intensive care unit: a randomized, double-blind controlled trial [ISRCTN43755713]. Crit Care. 2004;8(1):R1-R11.

20. Agarwal V, O’Neill PJ, Cotton BA, et al. Prevalence and risk factors for development of delirium in burn intensive care unit patients. J Burn Care Res. 2010;31(5):706-715.

21. Pandharipande P, Cotton BA, Shintani A, et al. Prevalence and risk factors for development of delirium in surgical and trauma intensive care unit patients. J Trauma. 2008;65(1):34-41.

22. Dubois MJ, Bergeron N, Dumont M, Dial S, Skrobik Y. Delirium in an intensive care unit: a study of risk factors. Intensive Care Med. 2001; 27(8):1297-1304

23. Marcantonio ER, Goldman L, Orav EJ, Cook EF, Lee TH. The association of intraoperative factors with the development of postoperative delirium. Am J Med. 1998;105(5):380-384.

24. Nelson BJ, Weinert CR, Bury CL, Marinelli WA, Gross CR. Intensive care unit drug use and subsequent quality of life in acute lung injury patients. Crit Care Med. 2000;28(11):3626-3630.

25. Ely EW, Truman B, Shintani A, et al. Monitoring sedation status over time in ICU patients: reliability and validity of the Richmond AgitationSedation Scale (RASS). JAMA. 2003;289(22):2983-2991.

26. Riker RR, Picard JT, Fraser GL. Prospective evaluation of the SedationAgitation Scale for adult critically ill patients. Crit Care Med. 1999; 27(7):1325-1329.

27. Botha JA, Mudholkar P. The effect of a sedation scale on ventilation hours, sedative, analgesic and inotropic use in an intensive care unit. Crit Care Resusc. 2004;6(4):253-257.

28. Morandi A, Jackson JC. Delirium in the intensive care unit: a review. Neurol Clin. 2011;29(4):749-763.

29. Cerejeira J, Firmino H, Vaz-Serra A, Mukaetova-Ladinska EB. The neuroinflammatory hypothesis of delirium. Acta Neuropathol. 2010; 119(6):737-754

30. Hshieh TT, Fong TG, Marcantonio ER, Inouye SK. Cholinergic deficiency hypothesis in delirium: a synthesis of current evidence. J Gerontol A Biol Sci Med Sci. 2008;63(7):764-772.

31. Pandharipande PP, Morandi A, Adams JR, et al. Plasma tryptophan and tyrosine levels are independent risk factors for delirium in critically ill patients. Intensive Care Med. 2009;35(11):1886-1892.

32. Ely EW, Shintani A, Truman B, et al. Delirium as a predictor of mortality in mechanically ventilated patients in the intensive care unit. JAMA. 2004;291(14):1753-1762.

33. Lin SM, Liu CY, Wang CH, et al. The impact of delirium on the survival of mechanically ventilated patients. Crit Care Med. 2004;32(11): 2254-2259.

34. Milbrandt EB, Deppen S, Harrison PL, et al. Costs associated with delirium in mechanically ventilated patients. Crit Care Med. 2004;32(4):955-962.

35. Pisani MA, Kong SY, Kasl SV, Murphy TE, Araujo KL, Van Ness PH. Days of delirium are associated with 1-year mortality in an older intensive care unit population. Am J Respir Crit Care Med. 2009; 180(11):1092-1097.

36. Shehabi Y, Riker RR, Bokesch PM, Wisemandle W, Shintani A, Ely EW. Delirium duration and mortality in lightly sedated, mechanically ventilated intensive care patients. Crit Care Med. 2010;38(12):2311-2318.

37. Bergeron N, Dubois MJ, Dumont M, Dial S, Skrobik Y. Intensive Care Delirium Screening Checklist: evaluation of a new screening tool. Intensive Care Med. 2001;27(5):859-864. 
38. Ely EW, Margolin R, Francis J, et al. Evaluation of delirium in critically ill patients: validation of the Confusion Assessment Method for the Intensive Care Unit (CAM-ICU). Crit Care Med. 2001;29(7): $1370-1379$.

39. Brook AD, Ahrens TS, Schaiff R, et al. Effect of a nursing-implemented sedation protocol on the duration of mechanical ventilation. Crit Care Med. 1999;27(12):2609-2615.

40. Brattebo G, Hofoss D, Flaatten H, Muri AK, Gjerde S, Plsek PE. Effect of a scoring system and protocol for sedation on duration of patients' need for ventilator support in a surgical intensive care unit. Qual Saf Health Care. 2004;13(3):203-205.

41. Sessler CN, Pedram S. Protocolized and target-based sedation and analgesia in the ICU. Crit Care Clin. 2009;25(3):489-513, viii.

42. Quenot JP, Ladoire S, Devoucoux F, et al. Effect of a nurse-implemented sedation protocol on the incidence of ventilator-associated pneumonia. Crit Care Med. 2007;35(9):2031-2036.

43. Kress JP, Pohlman AS, O'Connor MF, Hall JB. Daily interruption of sedative infusions in critically ill patients undergoing mechanical ventilation. $N$ Engl J Med. 2000;342(20):1471-1477.

44. Kress JP, Gehlbach B, Lacy M, Pliskin N, Pohlman AS, Hall JB. The long-term psychological effects of daily sedative interruption on critically ill patients. Am J Respir Crit Care Med. 2003;168(12):1457-1461.

45. Girard TD, Kress JP, Fuchs BD, et al. Efficacy and safety of a paired sedation and ventilator weaning protocol for mechanically ventilated patients in intensive care (Awakening and Breathing Controlled trial): a randomised controlled trial. Lancet. 2008;371(9607):126-134.

46. Jackson JC, Girard TD, Gordon SM, et al. Long-term cognitive and psychological outcomes in the awakening and breathing controlled trial. Am J Respir Crit Care Med. 2010;182(2):183-191.

47. Girard TD, Shintani AK, Jackson JC, et al. Risk factors for posttraumatic stress disorder symptoms following critical illness requiring mechanical ventilation: a prospective cohort study. Crit Care. 2007;11(1):R28.

48. Weinert CR, Sprenkle M. Post-ICU consequences of patient wakefulness and sedative exposure during mechanical ventilation. Intensive Care Med. 2008;34(1):82-90.

49. Granja C, Gomes E, Amaro A, et al. Understanding posttraumatic stress disorder-related symptoms after critical care: the early illness amnesia hypothesis. Crit Care Med. 2008;36(10):2801-2809.

50. Jones C, Griffiths RD, Humphris G, Skirrow PM. Memory, delusions, and the development of acute posttraumatic stress disorder-related symptoms after intensive care. Crit Care Med. 2001;29(3):573-580.

51. Larson MJ, Weaver LK, Hopkins RO. Cognitive sequelae in acute respiratory distress syndrome patients with and without recall of the intensive care unit. J Int Neuropsychol Soc. 2007;13(4):595-605.

52. Barr J, Egan TD, Sandoval NF, et al. Propofol dosing regimens for ICU sedation based upon an integrated pharmacokinetic-pharmacodynamic model. Anesthesiology. 2001;95(2):324-333.

53. Diedrich DA, Brown DR. Analytic reviews: propofol infusion syndrome in the ICU. J Intensive Care Med. 2011;26(2):59-72.

54. Fudickar A, Bein B. Propofol infusion syndrome: update of clinical manifestation and pathophysiology. Minerva Anestesiol. 2009;75(5): 339-344.

55. Tan JA, Ho KM. Use of dexmedetomidine as a sedative and analgesic agent in critically ill adult patients: a meta-analysis. Intensive Care Med. 2010;36(6):926-939.

56. Maze M, Scarfini C, Cavaliere F. New agents for sedation in the intensive care unit. Crit Care Clin. 2001;17(4):881-897.

57. Yahwak JA, Riker RR, Fraser GL, Subak-Sharpe S. Determination of a lorazepam dose threshold for using the osmol gap to monitor for propylene glycol toxicity. Pharmacotherapy. 2008;28(8):984-991.

58. Riker RR, Shehabi Y, Bokesch PM, et al. Dexmedetomidine vs midazolam for sedation of critically ill patients: a randomized trial. JAMA. 2009;301(5):489-499.

59. Pandharipande PP, Pun BT, Herr DL, et al. Effect of sedation with dexmedetomidine vs lorazepam on acute brain dysfunction in mechanically ventilated patients: the MENDS randomized controlled trial. JAMA. 2007;298(22):2644-2653.
60. Barrientos-Vega R, Mar Sánchez-Soria M, Morales-García C, Robas-Gómez A, Cuena-Boy R, Ayensa-Rincon A. Prolonged sedation of critically ill patients with midazolam or propofol: impact on weaning and costs. Crit Care Med. 1997;25(1):33-40.

61. Carson SS, Kress JP, Rodgers JE, et al. A randomized trial of intermittent lorazepam versus propofol with daily interruption in mechanically ventilated patients. Crit Care Med. 2006;34(5):1326-1332.

62. Walder B, Elia N, Henzi I, Romand JR, Tramer MR. A lack of evidence of superiority of propofol versus midazolam for sedation in mechanically ventilated critically ill patients: a qualitative and quantitative systematic review. Anesth Analg. 2001;92(4):975-983.

63. Mehta S, McCullagh I, Burry L. Current sedation practices: lessons learned from international surveys. Crit Care Clin. 2009;25(3): 471-488, vii-viii.

64. Rozendaal FW, Spronk PE, Snellen FF, et al. Remifentanil-propofol analgo-sedation shortens duration of ventilation and length of ICU stay compared to a conventional regimen: a centre randomised, cross-over, open-label study in the Netherlands. Intensive Care Med. 2009;35(2): 291-298.

65. Breen D, Karabinis A, Malbrain M, et al. Decreased duration of mechanical ventilation when comparing analgesia-based sedation using remifentanil with standard hypnotic-based sedation for up to 10 days in intensive care unit patients: a randomised trial [ISRCTN47583497]. Crit Care. 2005;9(3):R200-R210.

66. Karabinis A, Mandragos K, Stergiopoulos S, et al. Safety and efficacy of analgesia-based sedation with remifentanil versus standard hypnoticbased regimens in intensive care unit patients with brain injuries: a randomised, controlled trial [ISRCTN50308308]. Crit Care. 2004; 8(4):R268-R280.

67. Strom T, Martinussen T, Toft P. A protocol of no sedation for critically ill patients receiving mechanical ventilation: a randomised trial. Lancet. 2010;375(9713):475-480.

68. Cox CE, Reed SD, Govert JA, et al. Economic evaluation of propofol and lorazepam for critically ill patients undergoing mechanical ventilation. Crit Care Med. 2008;36(3):706-714.

69. Ho KM, Ng JY. The use of propofol for medium and long-term sedation in critically ill adult patients: a meta-analysis. Intensive Care Med. 2008;34(11):1969-1979.

70. Pandharipande PP, Sanders RD, Girard TD, et al. Effect of dexmedetomidine versus lorazepam on outcome in patients with sepsis: an a priori-designed analysis of the MENDS randomized controlled trial. Crit Care. 2010;14(2):R38.

71. Venn RM, Grounds RM. Comparison between dexmedetomidine and propofol for sedation in the intensive care unit: patient and clinician perceptions. Br J Anaesth. 2001;87(5):684-690.

72. Herr DL, Sum-Ping ST, England M. ICU sedation after coronary artery bypass graft surgery: dexmedetomidine-based versus propofol-based sedation regimens. $J$ Cardiothorac Vasc Anesth. 2003;17(5):576-584.

73. Jakob SM, Ruokonen E, Grounds RM, et al. Dexmedetomidine vs midazolam or propofol for sedation during prolonged mechanical ventilation: two randomized controlled trials. JAMA. 2012;307(11): 1151-1160.

74. Dasta JF, Kane-Gill SL, Pencina M, et al. A cost-minimization analysis of dexmedetomidine compared with midazolam for long-term sedation in the intensive care unit. Crit Care Med. 2010;38(2):497-503.

75. Baumgartner GR, Rowen RC. Clonidine vs chlordiazepoxide in the management of acute alcohol withdrawal syndrome. Arch Intern Med. 1987;147(7):1223-1226.

76. Cammarano WB, Pittet JF, Weitz S, Schlobohm RM, Marks JD. Acute withdrawal syndrome related to the administration of analgesic and sedative medications in adult intensive care unit patients. Crit Care Med. 1998;26(4):676-684.

77. Liatsi D, Tsapas B, Pampori S, Tsagourias M, Pneumatikos I, Matamis D. Respiratory, metabolic and hemodynamic effects of clonidine in ventilated patients presenting with withdrawal syndrome. Intensive Care Med. 2009;35(2):275-281. 
78. Neusy AJ, Lowenstein J. Blood pressure and blood pressure variability following withdrawal of propranolol and clonidine. J Clin Pharmacol. 1989;29(1):18-24.

79. Morandi A, Vasilevskis EE, Pandharipande PP, et al. Inappropriate medications in elderly ICU survivors: where to intervene? Arch Intern Med. 2011;171(11):1032-1034.

80. Laskowski K, Stirling A, McKay WP, Lim HJ. A systematic review of intravenous ketamine for postoperative analgesia. Can J Anaesth. 2011;58(10):911-923.

81. Hirota K, Lambert DG. Ketamine: new uses for an old drug? $\mathrm{Br} J$ Anaesth. 2011;107(2):123-126.

82. Martin J, Heymann A, Basell K, et al. Evidence and consensus-based German guidelines for the management of analgesia, sedation and delirium in intensive care - short version. Ger Med Sci. 2010;8: Doc02.

83. Kong KL, Willatts SM, Prys-Roberts C. Isoflurane compared with midazolam for sedation in the intensive care unit. BMJ. 1989;298(6683):1277-1280.

84. Mesnil M, Capdevila X, Bringuier S, et al. Long-term sedation in intensive care unit: a randomized comparison between inhaled sevoflurane and intravenous propofol or midazolam. Intensive Care Med. 2011;37(6):933-941.
85. Sackey PV, Martling CR, Nise G, Radell PJ. Ambient isoflurane pollution and isoflurane consumption during intensive care unit sedation with the Anesthetic Conserving Device. Crit Care Med. 2005;33(3): 585-590.

86. Papazian L, Forel JM, Gacouin A, et al. Neuromuscular blockers in early acute respiratory distress syndrome. N Engl J Med. 2010;363(12): 1107-1116.

87. Schweickert WD, Pohlman MC, Pohlman AS, et al. Early physical and occupational therapy in mechanically ventilated, critically ill patients: a randomised controlled trial. Lancet. 2009;373(9678):1874-1882.

88. Schweickert WD, Kress JP. Implementing early mobilization interventions in mechanically ventilated patients in the ICU. Chest. 2011;140(6):1612-1617.

89. Needham DM, Korupolu R, Zanni JM, et al. Early physical medicine and rehabilitation for patients with acute respiratory failure: a quality improvement project. Arch Phys Med Rehabil. 2010;91(4):536-542.

90. Vasilevskis EE, Pandharipande PP, Girard TD, Ely EW. A screening, prevention, and restoration model for saving the injured brain in intensive care unit survivors. Crit Care Med. 2010;38(Suppl 10): S683-S691.
Clinical Pharmacology: Advances and Applications

\section{Publish your work in this journal}

Clinical Pharmacology: Advances and Applications is an international, peer-reviewed, open access journal publishing original research, reports, reviews and commentaries on all areas of drug experience in humans. The manuscript management system is completely online and includes a very quick and fair peer-review system, which is all easy to use.

\section{Dovepress}

Visit http://www.dovepress.com/testimonials.php to read real quotes from published authors. 